\title{
Point-of-care and lung ultrasound incorporated in daily practice
}

\author{
E Neethling, ${ }^{1} \mathrm{MB}$ ChB, DA (SA), FCA (SA); F Roodt, ${ }^{1} \mathrm{MB}$ ChB, FCA (SA); C Beck, ${ }^{2} \mathrm{MB}$ ChB, DA (SA); \\ J L C Swanevelder, ${ }^{1} \mathrm{MB}$ ChB, DA (SA), FCA (SA), MMed (Anaes), FRCA \\ ${ }^{1}$ Department of Anaesthesia and Perioperative Medicine, Faculty of Health Sciences, Groote Schuur Hospital and University of Cape Town, \\ and Red Cross War Memorial Children's Hospital, Cape Town, South Africa \\ ${ }^{2}$ Department of Anaesthesia and Perioperative Care, Frere Hospital and Cecilia Makiwani Hospital, and Walter Sisulu University, \\ East London, South Africa
}

Corresponding author: J L C Swanevelder (justiaan.swanevelder@uct.ac.za)

\begin{abstract}
Point-of-care ultrasound (POCUS) is a fast-growing clinical utility and is becoming an essential clinical skill for all practitioners attending to critically ill patients. Ultrasound equipment is now smaller, more affordable and readily available in clinical work areas. POCUS is performed by a non-cardiologist physician at the patient's bedside as an adjunct to the physical examination. It is easily taught, non-invasive and allows for real-time clinical information. Bedside use of ultrasound imaging aids with rapid diagnosis of severe and life-threatening pathological conditions. It can be repeated, may change clinical management, and impact on patient outcome. POCUS has a broad clinical use, including, but not limited to, focused assessed transthoracic echocardiography (FATE), lung ultrasound imaging, extended focused assessment with sonography for trauma (e-FAST), vascular access and regional blocks. It may also be extended to detect endotracheal intubation and the estimation of intracranial pressure. Assessment of cardiac pathology by POCUS, performed by a novice examiner, has been shown to compare with the gold standard of an expert. Training is paramount. The physician should know his limitations and always relate the information back to the clinical scenario and context. By incorporating POCUS as part of our armamentarium and into our daily medical practice, we might see it reach its full clinical potential, optimising patient care and improving patient outcomes.
\end{abstract}

S Afr Med J 2018;108(5):376-381. DOI:10.7196/SAMJ.2018.v108i5.13313

Point-of-care ultrasound (POCUS) is a fast-growing clinical utility, and an essential clinical skill for all practitioners attending to patients in the perioperative period. It has been adopted in multiple areas of medicine and is fast becoming the standard of care, including but not limited to anaesthesia, surgery, intensive care, general medicine, emergency medicine and paediatrics. ${ }^{[1]}$

Bedside use of ultrasound imaging assists with rapid diagnosis of severe and life-threatening pathological conditions, and the effective management thereof. During the past decade, the development of new digital technology, miniaturisation of hand-held devices, affordability and increased availability of equipment have led to the introduction of this skill in everyday practice. Assessment of cardiac pathology by POCUS performed by a novice examiner is comparable to that of the gold standard specialist ultrasonographer ${ }^{[2]}$ In resourcepoor environments it has been adopted as a screening tool before patient referral to larger centres. In a recently published article in Heart by Ploutz et al., ${ }^{[3]}$ the authors used hand-held echo equipment to screen school-age children in Uganda for rheumatic heart disease. The study confirmed that non-expert findings correlated with expert review.

POCUS not only includes cardiac imaging, but also lung and abdominal ultrasonography, and is used when achieving vascular access.

Essential features of POCUS:

- simplified, limited scope

- goal and problem orientated

- time sensitive and repeatable

- qualitative

- performed by physician at the point of care

- adjuncts to physical examination.
When using ultrasound to examine the heart, it is important to distinguish between focused cardiac ultrasound (FOCUS), limited transthoracic echocardiography (TTE) and a comprehensive examination. ${ }^{[4]}$

A comprehensive TTE examination involves the use of specific equipment, assists with diagnosing pathology, and requires a larger knowledge base with specialised training. FOCUS as a screening modality has a more focused scope, and is used to answer a specific clinical question, often looking for a 'yes' or 'no' answer. This application does not require cardiology knowledge, and less intensive training is needed. ${ }^{[4]}$ However, interpretation of the images requires adequate training and is operator dependent. Clinical common sense is also needed to apply the additional echo findings to the clinical scenario. There is a well-known saying that ' $a$ fool with a stethoscope is also a fool with an echoprobe. FOCUS does not negate or replace the need for a formal diagnostic echocardiography investigation. Each has a distinct role and clinical use.

There are currently multiple different protocols, and acronyms abound, all available for training in POCUS: focused assessed transthoracic echocardiography (FATE), POCUS, extended focused assessment with sonography for trauma (e-FAST), FOCUS, emergency point-of-care ultrasound (EPCUS), bedside lung ultrasound in emergency (BLUE), internal medicine bedside ultrasound (IMBUS), rapid obstetric sonographic evaluation (ROSE), focused echocardiographic evaluation in life (FEEL) support, focused intensive care echocardiography (FICE), focused perioperative risk evaluation sonography involving gastro-abdominal haemodynamic and transthoracic ultrasound (FORESIGHT), and haemodynamic echocardiography examination in real time (HART). These different protocols all have a similar goal, i.e. to provide a structure to 
non-cardiologist practitioners to diagnose or confirm a specific clinical cardiorespiratory emergency that is responsible for patient haemodynamic instability.

POCUS is easily taught, ${ }^{[5]}$ non-invasive and readily available. In trained hands, it is the perfect diagnostic tool at the patient's bedside, outside the radiology department. ${ }^{[6]}$ POCUS provides real-time information that can guide clinical management, and should be used routinely in practice and training. ${ }^{[7]}$ POCUS should always be considered to complement the clinical evaluation, and the information must be related back to the clinical context.

\section{Probe selection}

In the emergency unit, theatre and intensive care unit, linear, curvilinear and phased-array probes are most commonly available. Probe selection is of critical importance in answering the relevant clinical question.

\section{Phased-array probe (1.5 - 2.0 MHz)}

This is most suitable for use in transthoracic echocardiography, and it can also be used for lung ultrasound to identify artefacts, but clarity of the lung image is not comparable to that of the curvilinear probe. ${ }^{[8]}$ Phased-array probes (Fig. 1) have a low frequency, with good penetration but poor resolution. Different sizes of probes are available. The normal adult size probe has a footprint area of $20 \times 14 \mathrm{~mm}$ (depending on the manufacturer). The correct footprint size should fit into the intercostal space, optimising the scanned area. In lung and cardiac ultrasonography a larger imaging depth is necessary.

\section{Curvilinear probe ( 3 - $5 \mathrm{MHz}$ )}

First introduced in the 1970s, this remains the probe of choice for abdominal ultrasound imaging (Fig. 2). The probe has a larger footprint than the phased-array probe, with a higher frequency, producing superior imaging in abdominal and lung ultrasound. The large footprint requires careful manipulation in the intercostal spaces, specifically posteriorly and laterally. ${ }^{[8]}$

\section{Straight linear probe $(8-12 \mathrm{MHz})$}

This probe is designed for imaging of superficial structures (Fig. 3). The higher frequencies provide better image resolution, but less penetration. The probe is ideal for intravascular line placement (central and peripheral), peripheral nerve blocks and superficial lung imaging to identify lung sliding. ${ }^{[8]}$

\section{Lung ultrasound}

There are different protocols adapted for lung ultrasound scans: BLUE - for the rapid diagnosis of life-threating respiratory failure, ${ }^{[9]}$ and fluid administration limited by lung sonography (FALLS).

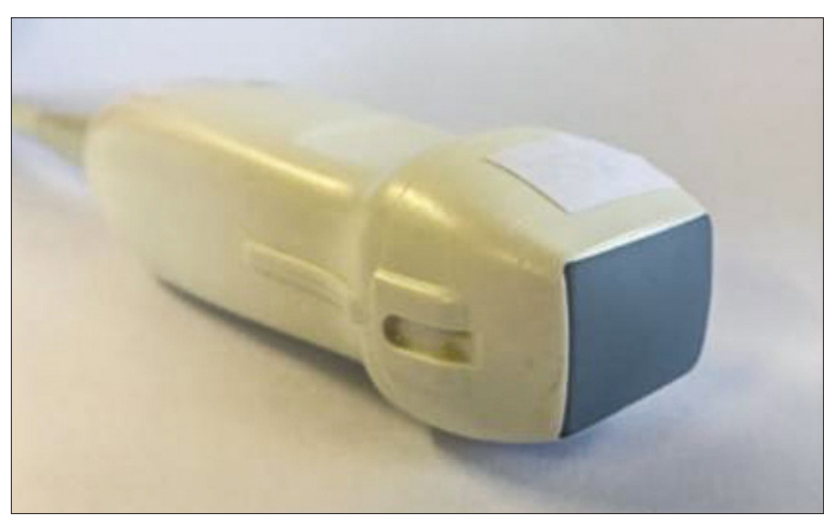

Fig. 1. Phased-array probe.

\section{Positioning of the patient}

The ideal position of the patient is supine, with head elevated $30-45^{\circ}$ (Fig. 4). The anterior chest wall is divided into 8 zones (4 left and 4 right). The rules of gravity apply; air (pneumothorax) accumulates

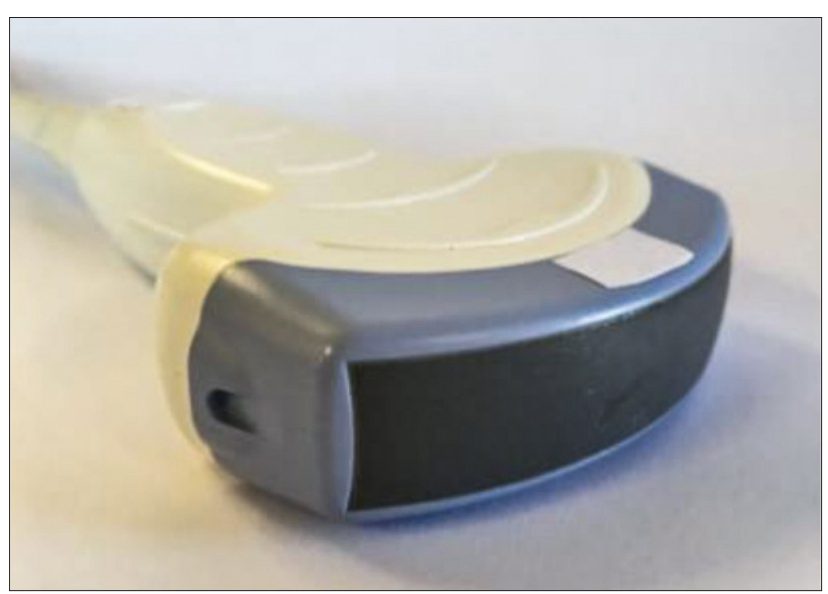

Fig. 2. Curvilinear probe.

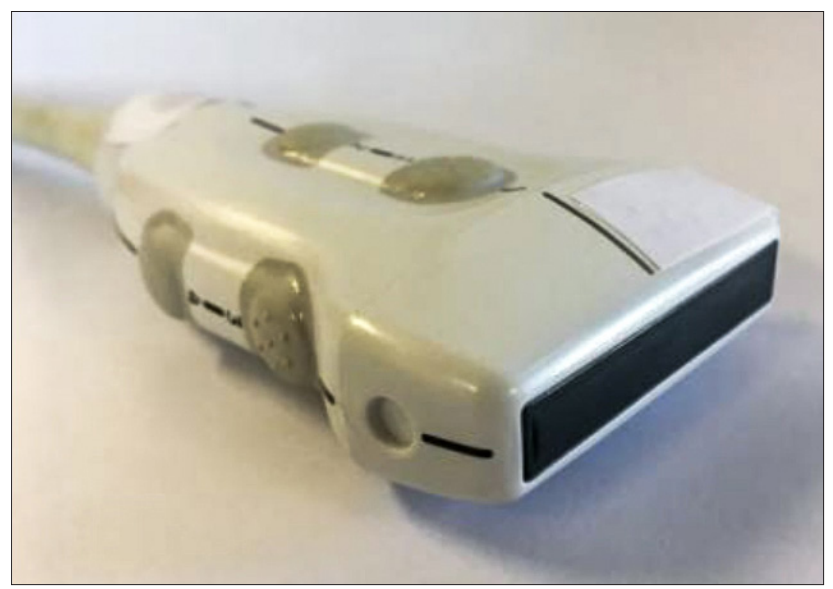

Fig. 3. Linear probe.

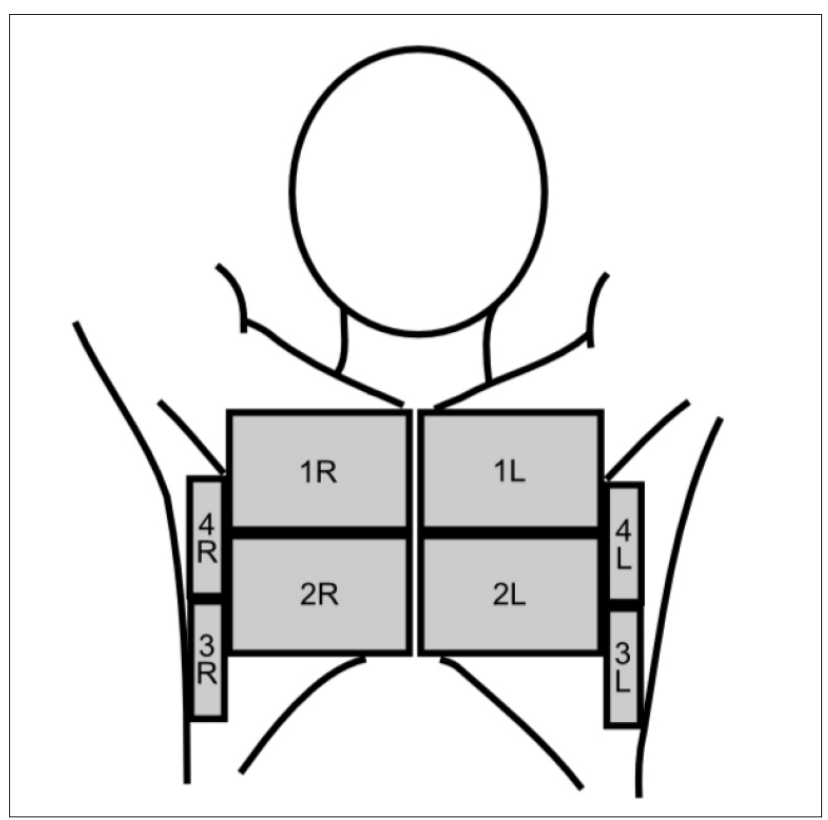

Fig. 4. Anterior and lateral lung zones. (Prof. Erik Sloth and USABCD.org, with permission.) 
in the least dependent part of the chest, and fluid in the most dependent part.

\section{Normal anatomy visualised}

During lung ultrasound, it is important to remember that the assessment is based on artefact identification and real-time image assessment.

\section{Lung sliding}

The normal anatomy of the pleural cavity consists of two pleural layers. The parietal pleura is adherent to the inner surface of the thoracic cavity and the visceral pleura is a delicate membrane covering the underlying lung tissue. The space between the pleurae is normally closely opposed with a small film of serous fluid. During normal respiration, the pleurae slide over each other. The artefact caused by this phenomenon can be appreciated as lung sliding. It may appear as a line of 'marching ants', with a line of black and white dots moving to and fro.

The absence of lung sliding may occur in: Any condition causing absent ventilation of the lung being investigated

- pneumonectomy

- inadvertent intubation of the other bronchus

- lung isolation during single lung ventilation

- bullous lung disease

- apnoea.

Abnormal adherence or absence of the parietal and visceral pleura

- pneumothorax

- pleural effusion

- pleurectomy/pleurodesis

- massive atelectasis

- acute respiratory disress syndrome

- pneumonia.

Common reasons for absent lung sliding in trauma:

- apnoea

- pneumothorax

- selective bronchus intubation.

\section{The Bat sign}

This was originally described by Lichtenstein ${ }^{[15]}$ to correctly identify the pleura. The intercostal ribs appear as the wings of a bat and the pleural line represents the bat's body.

\section{A-lines}

These lines are seen inside the space and represent reverberations of the pleural line (Fig. 5A). They are horizontal with the pleural line and motionless. They may be complete or incomplete and occur owing to the presence of air below the pleural line.

\section{B-lines}

The B-lines are formed owing to the presence of fluid in the interstitial space, and are hyperechoic reverberation artefacts (Fig. 5B). They are vertical in nature, start at the pleural line, span the entire depth of the image, move with normal respiration and erase the appearance of A-lines. They remind one of 'Hollywood lights' or comet tails. The appearance of B-lines can be normal or abnormal, indicating the presence of pulmonary interstitial syndrome. The presence of occasional B-lines (>2), especially in the dependent bases, can be considered normal. The presence of $>3$ lines in $>2$ zones per side defines the diagnosis of pulmonary interstitial syndrome. ${ }^{[10]}$

\section{Motion-mode assessment}

Motion mode (M-mode) examines the change/movement of structures on a chosen ultrasound line over time. If lung sliding is present, the image would symbolise a seashore. The 'sand' represents normal lung sliding (Fig. 6), created by a motion artefact, while the 'sea and rolling waves' represent

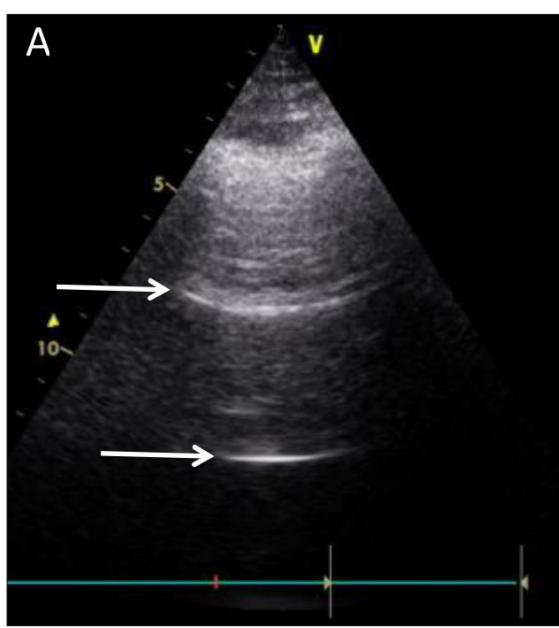

the subcutaneous tissue. If lung sliding is absent (Fig. 7), there is no motion artefact; therefore, only horizontal straight lines can be visualised. It is similar to a barcode or is also referred to as the stratosphere sign ${ }^{[9]}$ This may indicate the presence of a pneumothorax.

\section{Lung pulse}

Movement of the pleural line vertically in synchrony with the cardiac rhythm is called the lung pulse. This is normally transmitted through a consolidated area and is useful in distinguishing pneumothorax from consolidation. ${ }^{[1]}$ The presence of this sign rules out the diagnosis of a pneumothorax.

\section{Clinical applications}

The identification and interpretation of the signs are operator dependent and require adequate training in image acquisition and interpretation.

\section{Pneumothorax}

Ultrasonography identification of a pneumothorax has a higher sensitivity than chest X-ray identification. ${ }^{[12]}$

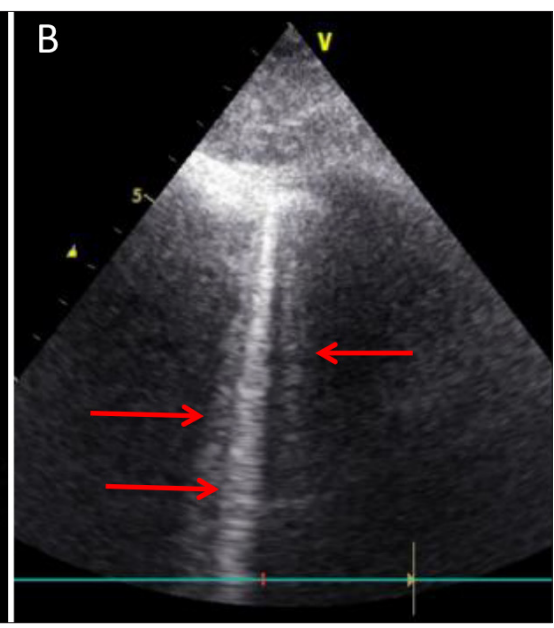

Fig. 5. Examples of lung ultrasound findings. (A) Hyperechoic horizontal artefacts arising from the pleural line (A-lines) (white arrows). The presence of A-lines in lung ultrasound imaging indicates normal lung insterstitium. (B) Hyperechoic reverberation artefacts arising from the pleural line to the bottom of the screen (B-lines) (red arrows). The presence of B-lines in lung ultrasound imaging indicates increased fluid contents in lung interstitium.
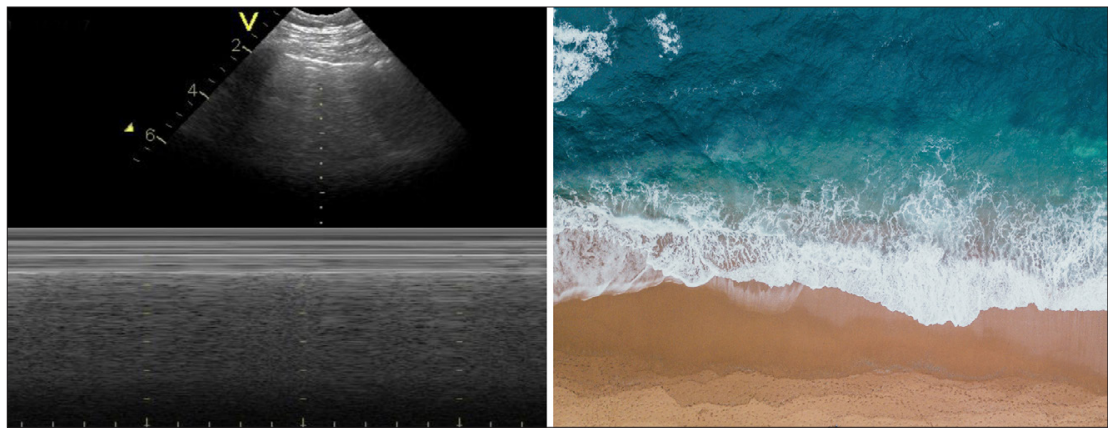

Fig. 6. Motion mode: normal lung sliding visualised as the seashore sign. (Prof. Erik Sloth and USABCD.org, with permission.) 
Assessment should start at the least dependant areas of the lung. The initial plane of assessment is longitudinal with the long axis of the body; placement of the probe should be between the parasternal and mid-clavicular line. The probe is then moved towards the dependent lateral aspect of the chest, assessing all 4 anterior lung zones on the left and right side of the chest. At each point assess for: lung sliding, B-line pattern and lung pulse. ${ }^{[1]}$ The absence of one or more of these may indicate the possibility of a pneumothorax.

\section{Lung point}

Identification of the lung point is $100 \%$ specific in diagnosing a pneumothorax (Fig. 8). ${ }^{[13]}$ If there is absence of lung sliding and a B-line pattern in the anterior chest, movement of the probe laterally to the dependent part of the lung may identify this

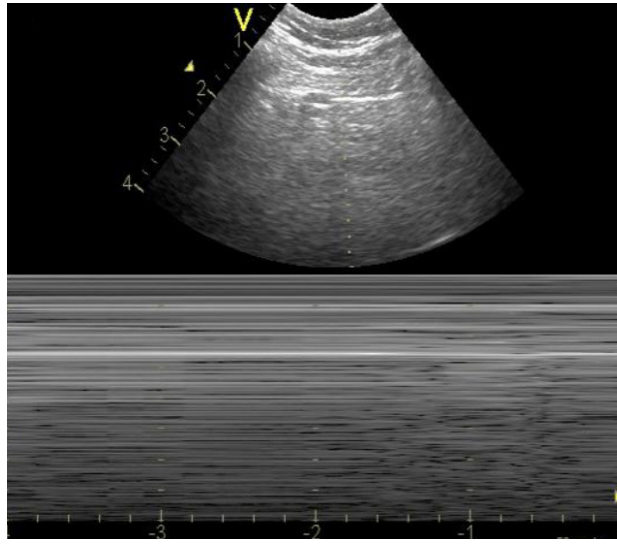

point as where normal sliding of the lung is replaced by the pneumothorax. ${ }^{[13]}$

\section{Pleural effusion}

The sensitivity and specificity of ultrasound as a modality to identify pleural effusion approaches $100 \%$. Ultrasound imaging can detect as little as $5-20 \mathrm{~mL}$ fluid in the pleural space. Chest radiography requires a minimum amount of $200-300 \mathrm{~mL}$ fluid in the pleural space to obliterate the costophrenic angle. ${ }^{[14]}$ Ultrasound imaging also assists with the differentiation between pleural thickening and pleural fluid accumulation, and may assist in differentiating a transudate from an exudate.

\section{Positioning}

The patient is positioned in a sitting or semiFowler position. The rules of gravity apply and therefore fluid accumulates in the dependent

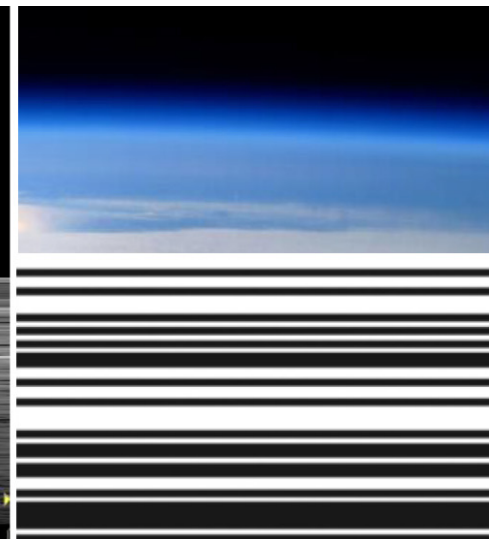

Fig. 7. Motion mode: absent lung sliding visualised as the barcode and stratosphere sign. (Prof. Erik Sloth and USABCD.org, with permission.)

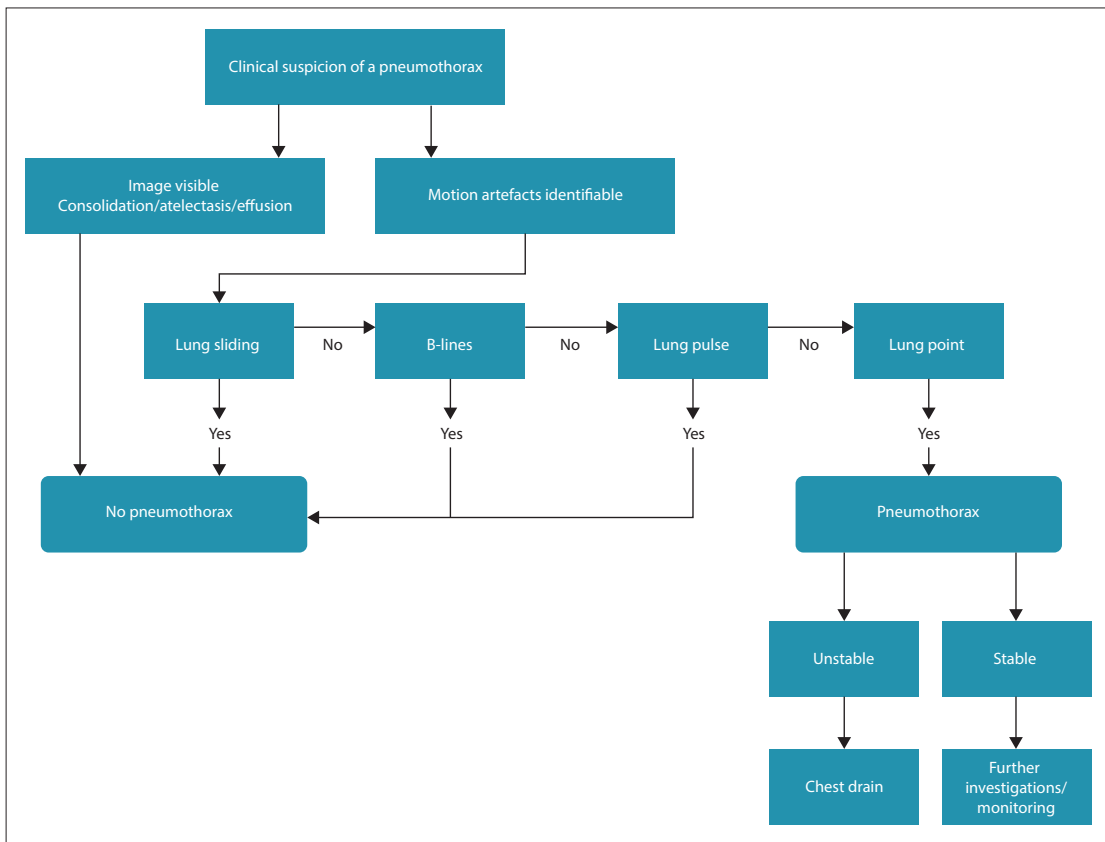

Fig. 8. Algorithm for identification of a pneumothorax. parts of the lung. The sitting position is used to assess the size of a pleural effusion.

\section{Normal anatomy}

Chest wall, hemidiaphragm, liver (right) and spleen (left) can be identified. If there is no fluid in the pleural space, the hemidiaphragm appears as though the image is 'wiped off' the screen during the normal inspiration.

\section{Signs on ultrasound}

Quad sign

This is a static sonographic image observed in the presence of a pleural effusion. The image demonstrates four distinct lines, which represent the lung, parietal pleura, rib and fluid. This sign is classically seen when a smaller pleural effusion is present, with the fluid appearing anechoic. ${ }^{[9]}$

\section{Sinusoid sign}

By placing M-mode through the effusion, the free movement of lung line towards pleural line can be seen. The movement of visceral pleura during inspiration and expiration creates the sinusoidal waveform, which indicates the presence of a pleural effusion. ${ }^{[14]}$

\section{Determining the size of a pleural effusion} Numerous methods and formulas have been described to quantify the size of a pleural effusion. Estimation of an accurate volume remains difficult. As a rule of thumb any effusion $>4 \mathrm{~cm}$ will measure $>1000 \mathrm{~mL}^{[14]}$

Volume of pleural effusion $=($ distance between visceral and parietal pleura in $\mathrm{mm}$ ) $\times 20$. The measurement is end-expiratory and measured as the maximum distance between pleura. ${ }^{[15]}$

\section{Differentiating transudate from exudate} Traditionally, the differentiation is done biochemically using Light's criteria. With the use of ultrasound, the nature of the image may suggest a possible diagnosis. Azam et al. ${ }^{[16]}$ proposed a simple scoring system, awarding each finding with 1 point. Bilateral pleural effusions, absence of loculated fluid, anechoic fluid, non-thickened pleura, congested liver and non-collapsing inferior vena cava each scores 1 point. A score of $>4$ is highly sensitive and specific for the diagnosis of a transudate. ${ }^{[16]}$

\section{Alveolar syndromes}

Alveolar consolidations are easily visualised. Most consolidated areas reach the pleural surface and can therefore be assessed by ultrasound imaging. The consolidation appears hypoechoic or anechoic and is therefore 
easily distinguishable from normally aerated lung that appears hyperechoic. ${ }^{[18]}$

\section{Sonographic signs of consolidation Hepatised lung}

If the lung is highly fluid filled, it resembles tissue in echogenicity. If the consolidation is right sided, the lung resembles the liver. If the area of consolidation is left sided, it will resemble the spleen.

\section{Presence of a bronchogram}

A bronchogram refers to the phenomenon of visible bronchi. Bronchi become visible owing to surrounding fluid-filled alveoli. Within areas of consolidation, these can be seen as hyperechoic areas. Air bronchograms have a white appearance, while fluid bronchograms have a hypoechoic appearance and are specific for the diagnosis of pneumonia.

\section{Limitations in performing focused lung ultrasound imaging}

Limitations in performing focused lung ultrasound imaging are as follows:

- it is an extension of the physical examination

- clinician should know his/her limitations

- inherent to the techniques

- individual level of skill

- training and experience

- neither comprehensive nor qualitative assessment

- pattern recognition of major/life-threatening pathology

- training is of the utmost importance.

\section{Point-of-care thoracic} echocardiography

The suggested targets for a focused POCUS according to the international consensus statement are the following: ${ }^{[19]}$

- left ventricular dimensions and systolic function

- right ventricular dimensions and systolic function

- volume status

- pericardial effusion and tamponade physiology

- gross signs of chronic heart disease

- gross valvular abnormalities

- large intracardiac masses.

\section{Indications for POCUS}

Indications for POCUS are the following:

- haemodynamic instability or undifferentiated shock

- cardiac arrest

- pericardial effusion/tamponade

- heart failure

- high-risk cardiac patients.

A full review of transthoracic echocardiography is beyond the scope of this article. Numerous protocols have been developed for use in point-of-care thoracic echocardiography.

All of the protocols focus on four basic cardiac views:

- parasternal short axis

- parasternal long axis

- subcostal 4-chamber view

- apical 4-chamber view.

The following views are used to answer specific questions: Subcostal and apical 4-chamber views

- biventricular size and function
- atrial size

- obvious valvular pathology

- pericardial effusion

- cardiac motion during cardiac arrest/resuscitation (subcostal view).

Parasternal short-axis views

- 'birds-eye' view of biventricular size and function

- presence of pericardial effusion

- left ventricular filling

- regional wall motion abnormalities.

Parasternal long-axis view

- left ventricular size and function

- mitral and aortic valves

- right ventricular size

- presence of pericardial and left pleural effusions.

\section{Abdominal point-of-care ultrasound}

The main application of abdominal POCUS is the detection of free abdominal fluid in the patient in the trauma and medical emergency unit. Ultrasound is the ideal tool for the rapid assessment of the haemodynamically unstable patient when the use of other diagnostic modalities, such as abdominal computed tomography (CT), is not practical. A full review of abdominal ultrasound is beyond the scope of this article.

The four basic views are:

- subcostal window

- identification of haemopericardium, which appears as a dark anechoic stripe

- right upper quadrant

- evaluate for fluid in the pleura, subphrenic and hepatorenal areas (Morison's pouch) and around the kidney

- left upper quadrant

- evaluate for fluid in the pleural, subphrenic and splenic areas and around the kidney

- pelvic transverse and longitudinal views

- assessment for fluid collection behind the bladder.

Aetiology of spontaneous haemoperitoneum in the non-trauma setting can vary and causes can be classified as follows: ${ }^{[20]}$

- hepatic

- splenic

- vascular (aneurysmal)

- gynaecological

- coagulopathy.

\section{Ultrasound for vascullar access}

Central venous catheters are routinely used in anaesthesiology, the intensive care unit, trauma unit and radiology suite. Complications arising from the procedure, such as inadvertent arterial puncture, haemothorax, pneumothorax and haematoma, can be life threatening. A recent Cochrane review, ${ }^{[21]}$ comparing ultrasound guidance with the landmark technique for the placement of internal jugular venous catheterisation, found improved safety and a decreased number of attempts when ultrasound is used.

\section{Systematic approach to ultrasound-guided vascular access}

A systematic approach to ultrasound-guided vascular access is as follows: ${ }^{[2]}$

- identify anatomy and site of insertion

- confirm arterial (pulsatile, non-compressible) v. venous (compressible/ collapsible) anatomy 
- ensure patency of desired vein/artery

- real-time ultrasound imaging, in-plane or out-of-plane technique with visualisation of cannula

- confirm guide wire position in desired vein prior to dilation.

The use of ultrasound for vascular access is not limited to only central venous access, but is now common in the angiography suite to improve safety and decrease complications during arterial puncture. It is also an indispensible tool in patients with difficult peripheral venous access.

\section{Conclusion}

POCUS is a fast-growing clinical modality and becoming an essential skill for all physicians dealing with potentially unstable patients. It allows rapid evaluation in life-threatening clinical scenarios. Imaging is performed by the caring physician at the patient's bedside to answer a specific clinical question. It improves patient safety, prevents complications, allows rapid treatment and improves diagnostic accuracy. It is easily taught and can be used effectively in a variety of clinical arenas and situations. It is, however, imperative to understand that POCUS is a screening tool and does not replace formal imaging techniques.

Adequate training is paramount to ensure patient safety, as this modality is operator dependent. The caring physician should know his/her limitations, always relate the information back to the clinical scenario, and communicate with experts and other specialists in the field. The physician should keep up to date with the development of different techniques and incorporate those in daily practice to improve accuracy of the imaging interpretation. Incorporating POCUS into daily medical practice has a major impact on the outcome of haemodynamically unstable patients.

Acknowledgements. None.

Author contributions. EN: literature review and manuscript preparation. FR: literature review and manuscript preparation. CB: manuscript preparation. JLCS: literature review, manuscript preparation and senior author.

Funding. None.

Conflicts of interest. None.

1. Andronikou S, Sergot L. 'Point-of-care ultrasound' - legitimate terminology. Pediatr Radiol 2017;47(13):1849 1850. htips//doi.org/10.1007/500247-017-3978-7
2. Frederiksen CA, Juhl-Olsen P, Andersen NH, et al. Assessment of cardiac pathology by point-of-care Itrasonography performed by a novice examiner is comparable to the gold standard. Scand J Trauma Resusc Emerg Med 2013:21:87.

3. Ploutz M, Lu JC, Scheel J, et al. Handheld echocardiographic screening for rheumatic heart disease by non-experts. Heart 2016;102(1):35-39. https://doi.org/10.1136/heartjnl-2015-308236

4. Coker BJ, Zimmerman JM. Why anesthesiologists must incorporate focused cardiac ultrasound into 4. Coker BJ, Zimmerman JM. Why anesthesiologists must incorporate focused cardiac ultrasound in
daily practice. Anesth Analg 2017;124(3):761-765. https://doi.org/10.1213/ANE.0000000000001854 5. Heiberg J, Hansen LS, Wemmelund K, et al. Point-of-care clinical ultrasound for medical students. Heiberg , Hansen LS, Wemmelund K, et al. Point-of-care clinical ultrasound
Ultrasound Int Open 2015;1(2):E58-E66. https://doi.org/10.1055/s-0035-1565173

6. Enghard P, Rademacher S, Nee J, et al. Simplified lung ultrasound protocol shows excellent prediction of extravascular lung water in ventilated intensive care patients. Crit Care 2015;19:36. https://doi. org/10.1186/s13054-015-0756-

7. Ramsingh D, Gudzenko V, Martin RD. Point-of-care ultrasound: Novel technology to routine perioperative assessment tool. Anesth Analg 2017;124(3):709-711. https://doi.org/10.1213/ANE. 0000000000001529

8. Szabo TL, Lewin PA. Ultrasound transducer selection in clinical imaging practice. J Ultrasound Med 2013;32(4):573-582. https://doi.org/10.7863/jum.2013.32.4.573

9. Lichtenstein DA. BLUE-protocol and FALLS-protocol: Two applications of lung ultrasound in the critically ill. Chest 2015;147(6):1659-1670. https://doi.org/10.1378/chest.14-1313

0. Volpicelli G, Elbarbary M, Blaivas M, et al. International evidence-based recommendations for point-ofcare lung ultrasound. Intens Care Med 2012;38(4):577-591. https://doi.org/10.1007/s00134-012-2513-4 Volpicelli G. Sonographic diagnosis of pneumothorax. Intens Care Med 2011;37(2):224-232. https://doi. org/10.1007/s00134-010-2079-

12. Vafaei A, Hatamabadi HR, Heidary K, et al. Diagnostic accuracy of ultrasonography and radiography in initial evaluation of chest trauma patients. Emerg (Tehran) 2016;4(1):29-33.

13. Lichtenstein D, Meziere G, Biderman P, et al. The 'lung point': An ultrasound sign specific to pneumothorax. Intens Care Med 2000;26(10):1434-1440. https://doi.org/10.1007/s001340000627

14. Capper SI, Ross IJ, Sandstrom E, Braidley PC, Morgan-Hughes NJ. Transoesophageal echocardiography for the detection and quantification of pleural fluid in cardiac surgical patients. $\mathrm{Br} J$ Anaesth 2007;98(4):442-446. https://doi.org/10.1093/bja/aem010

15. Lichtenstein D. Lung ultrasound in the critically ill. Curr Opin Crit Care 2014;20(3):315-322. https://doi. org/10.1097/MCC 0000000000000096

16. Azam SM, Owen WT, Kamalanathan M, et al. A simple score based on ultrasound criteria to distinguish between exudative vs. transudative pleural effusion. Eur Resp J 2014;44(Suppl 58):666.

17. Balik $M$, Plasil P, Waldauf $\mathrm{P}$, et al. Ultrasound estimation of volume of pleural fluid in mechanically Balik M, Plasil P, Waldauf P, et al. Ultrasound estimation of volume of pleural fluid in mech
ventilated patients. Intens Care Med 2006;32(2):318. https://doi.org/10.1007/s00134-005-0024-2

18. Volpicelli G, Silva F, Radeos M. Real-time lung ultrasound for the diagnosis of alveolar consolidation and interstitial syndrome in the emergency department. Eur I Emerg Med 2010;17(2):63-72.

19. Via G, Hussain A, Wells M, et al. International evidence-based recommendations for focused cardiac ultrasound. J Am Soc Echocardiogr 2014;27(7):el-e33. https://doi.org/10.1016/j.echo.2014.05.001

20. Lucey BC, Varghese JC, Anderson SW, et al. Spontaneous hemoperitoneum: A bloody mess. Emerg Radiol 2007;14(2):65-75

21. Brass P, Hellmich M, Kolodziej L, et al. Ultrasound guidance versus anatomical landmarks for internal jugular vein catheterization. Cochrane Database Syst Rev 2015;(1):CD006962. https://doi. org/10.1002/14651858.CD006962.pub2

22. Saugel B, Scheeren TWL, Teboul JL. Ultrasound-guided central venous catheter placement: A structured review and recommendations for clinical practice. Crit Care 2017;21(1):225. https://doi.org/10.1186/ s13054-017-1814-y

23. Zimmerman $\mathrm{JM}$, Coker BJ. The nuts and bolts of performing focused cardiovascular ultrasound (FoCUS). Anesth Analg 2017;124(3):753-760. https://doi.org/10.1213/ANE0000000000001861

\section{Additional free and downloadable resources}

FATE card for android or iOS devices

Virtual transthoracic echocardiography Toronto - www.pie.med.utoronto.ca

www.echopedia.org

www.123sonography.com

Accepted 3 April 2018 